\title{
Evaluación de la indicación, consumo y costos de antifúngicos en un hospital pediátrico de Chile
}

\author{
Marlon Barraza', Natalia Barnafi, Guillermo Ortiz², Juan PabloTorres², \\ Paulina Coria², Paula Catalán ${ }^{3}$, Julia Palma y Jorge Morales ${ }^{3}$
}

\section{Evaluation of the prescription, consumption and costs of antifungal drugs in a pediatric hospital in Chile}

Background: The increase of invasive fungal disease (IFD) in immunocompromised patients has led to the frequent prescription of highly active antifungal drugs but with a high economic cost. Aim: To characterize the use of antifungals drugs, evaluate its prescription and determine consumption and associated costs. Methods: Retrospective descriptive study from January 2015 to April 2016. Audit of prescriptions and review of clinical files. Each prescription was classified according to whether it corresponded to a possible, probable or proven invasive fungal disease (IFD). Consumptions and treatment costs were calculated. Results: 152 antifungal prescriptions were audited in 79 patients. The total cost of antifungal medications was US $\$ 714,413.52 .1 \%$ of the expenditure (US \$ 372,319) corresponded to indications in proven IFD, $10.7 \%$ (US \$ 76,377) probable IFD, $0.8 \%$ (US \$ 5,638 ) non-IFI, $12.2 \%$ (US \$ 87,459) IFD possible and 1.5\% (US \$10,896) non-IFD and 22.6\% (US \$ 161,723) was prophylaxis. The highest consumption was in indications related to IFD tested with a proven DOT of 10.54 days, with liposomal amphotericin B and iv voriconazole the drugs with the highest consumption with a DOT probable_AnBL of 3.15 and DOT proven voriconazole iv of 3.01. Conclusions: The consumption of antifungal drug medications generates high costs at $12 \%$ of the total pharmacy budget of our institution. The expense was associated mainly with the indications in IFI tested the voriconazole and amphotericin B liposomal with the highest consumption which added to its high cost and prolonged days of general therapy a big impact in the budget.

Key words: Antifungal drugs; antifungal stewardship; economic impact; invasive fungal diseases.

Palabras clave: Enfermedad fúngica invasora; antifúngicos; antifungal stewardship; impacto económico.

\section{Introducción}

L

a enfermedad fúngica invasora (EFI) es una de las principales causas de mortalidad en los pa-

cientes hospitalizados ${ }^{1}$. La frecuencia de las EFI ha aumentado en las dos últimas décadas, lo que está estrechamente relacionado con el aumento del número de pacientes inmunocomprometidos con riesgo de infección oportunista, como hemato-oncológicos (HO), receptores de trasplante de precursores hematopoyéticos (TPH), receptores de trasplante de órganos sólidos (TOS), otros pacientes inmunocomprometidos y aquellos con cirugía mayor, especialmente de intestino ${ }^{2}$. En Chile, según datos del Programa Nacional de Drogas Antineoplásicas Infantil (PINDA), la incidencia de EFI en la población oncológica pediátrica que cursa un episodio de neutropenia y fiebre es de $9 \%$, siendo la candidiasis la EFI más frecuente en este grupo de pacientes ${ }^{3}$.

El diagnóstico e inicio tardío de la terapia antifúngica apropiada para hongos de los géneros Candida y Aspergillus, del orden Mucorales y Pneumocystis jiroveci, se asocian con peores resultados clínicos.
El diagnóstico de EFI es difícil dado la presentación clínica inespecífica, la baja sensibilidad de los cultivos, la microscopia y los exámenes histológicos, entre otros.

La necesidad de inicio precoz de la terapia ha contribuido al uso excesivo de antifúngicos, donde su uso se basa en riesgos y no certezas ${ }^{2-4}$. Varios estudios han podido establecer el aumento en el consumo y en algunos casos el uso inadecuado de las terapias antifúngicas ${ }^{5-7}$. Esto se ha convertido en un importante problema de salud, ya que está relacionado con la exposición innecesaria a estos fármacos, generando un aumento en las reacciones adversas, las interacciones farmacológicas y un aumento general de la resistencia antifúngica.

Por otro lado, la aparición de nuevos fármacos y/o formulaciones como azoles, equinocandinas y anfotericina B liposomal (AnB-L) con amplio espectro, que proporcionan un buen margen de seguridad y efectividad, ha contribuido al aumento del uso de estas terapias ${ }^{2}$.

Los fármacos antifúngicos sistémicos son de alto valor económico y, por lo general, se recetan durante largos períodos de tiempo, lo que resulta en un aumento de los costos, tanto para las instituciones como para los
Hospital Dr. Luis Calvo Mackenna. Santiago, Chile. Unidad de Farmacia, Dr. Luis Calvo Mackenna hospital. 2Departamento de Pediatría y Cirugía Infantil, Infectología Universidad de Chile. Unidad de Trasplante de Médula Ósea, Hospital Dr. Luis Calvo Mackenna.

Los autores declaran no tener conflicto o interés financiero en cualquier producto o servicio mencionado en el manuscrito, incluyendo subvenciones, equipo medicamentos, empleo, regalos y honorarios.

Recibido: 22 de diciembre de 2017

Aceptado: 7 de junio de 2018

Correspondencia a: Marlon Barraza Olivares mbarraza@calvomackenna.cl 
pacientes. Los datos internacionales indican que el mercado de antifúngicos sistémicos aumentó de US \$ 2.100 millones en 1999 a US \$ 3.300 millones en 2003 y los azoles dominan el mercado con $52 \%$ de las ventas en el año $2003^{8}$. Un estudio efectuado en nuestro hospital estimó que el costo neto atribuible al tratamiento de la aspergilosis invasora fue de $\$ 13.274 .185$ pesos chilenos (US \$ 23.600) ${ }^{9}$.

Este aumento en la utilización de antifúngicos, sumado a su alto costo, ha llevado a la necesidad de promover programas de uso apropiado de antifúngico conocidos como "antifungal stewardship", lo que hasta la fecha se ha traducido en una reducción en el uso y gasto de antifúngicos, sin cambios en mortalidad ${ }^{4,10}$.

En nuestra institución, el uso de antifúngicos está condicionado a la evaluación clínica y posterior autorización, mediante formularios de medicamentos de uso restringido, por el equipo de infectología y monitorizado por el servicio de farmacia del hospital.

El objetivo de este estudio fue caracterizar el uso de antifúngicos, evaluar su indicación y determinar el consumo y costos asociados a estas terapias.

\section{Metodología}

Se realizó un estudio descriptivo, retrospectivo, desde enero de 2015 a abril de 2016 en el Hospital Dr. Luis Calvo Mackenna, hospital pediátrico de alta complejidad de Santiago de Chile. Se realizó una auditoría en la unidad de farmacia de las prescripciones de antifúngicos de uso restringido y se incluyeron todas aquellas asociadas a antifúngicos de alto costo: voriconazol intravenoso y oral, AnB-L, anidulafungina, caspofungina y posaconazol oral. Se excluyó el uso de fluconazol, debido a que no es el fármaco de primera línea en EFI en pacientes inmunocomprometidos.

Se revisaron las fichas clínicas de pacientes a quienes se les prescribieron antifúngicos de alto costo y se obtuvieron datos demográficos como edad, sexo, peso, diagnóstico, microorganismo aislado en algunos casos y dosis empleada del fármaco en estudio. Cada prescripción de antifúngico se clasificó de acuerdo a si estaba relacionada a un episodio de EFI probada, probable o posible de acuerdo con el consenso EORTC/MSG 2002 (revisado en 2008) ${ }^{11}$. Además, para los episodios que no calzaban dentro de esta categorización, se designaron las categorías de EFI descartada después de un estudio completo con microbiología, imágenes y pruebas serológicas o moleculares negativas y las infecciones superficiales por hongos, no invasoras, fueron clasificadas como no$E F I$. Por otra parte, cada indicación de antifúngico se clasificó de acuerdo a si correspondió a un tratamiento dirigido (ajustado a microbiología) si se logró identificar el microorganismo causal, empírico si la utilización del antifúngico se basó en criterios clínicos sugerentes de etiología probable, pero sin la certeza microbiológica y profiláctico, si se utilizó como prevención secundaria por haber presentado una EFI previa que justificara el uso de estos fármacos. (En nuestra institución se utiliza profilaxis primaria, sólo en pacientes TPH, con fluconazol, el que no fue considerado en este estudio).

Se evaluó el consumo de antifúngico en cada clasificación; para esto se calculó la medida DOT (del inglés days of therapy = días de terapia), que corresponde a los días de tratamiento por 100 pacientes días. Una DOT representa la administración de un solo fármaco en un día dado, independientemente del número de dosis administradas o de la dosificación, lo que la hace ser la unidad recomendada para pediatría ya que en general las dosis terapéuticas en este grupo de pacientes se calculan basándose en el peso o área de superficie corporal ${ }^{12,13}$. El costo asociado a cada episodio se obtuvo a través del departamento de farmacia, utilizando el costo actual (diciembre de 2016) de cada medicamento obtenido del Sistema de Administración y Manejo Hospitalario (SAM).

\section{Análisis estadístico}

Las variables continuas se representaron como media \pm desviación estándar y mediana \pm intervalo. Las variables cualitativas se expresaron como porcentajes. Para el análisis estadístico, se utilizó la versión Microsoft ${ }^{\circledR}$ Excel $^{\circledR}$ 2011 para Mac OS versión 14.2.0.

\section{Resultados}

Se auditaron 152 prescripciones de antifúngicos de alto costo, correspondientes a 79 pacientes con episodios de EFI cuyas características se resumen en la Tabla 1.

Los fármacos más prescritos en el total de episodios de EFI fueron equinocandinas: anidulafungina 26,3\% ( $\mathrm{n}=40$ prescripciones) y caspofungina $12,5 \%(\mathrm{n}=19$ prescripciones), seguidos de voriconazol intravenoso $25,7 \%(\mathrm{n}=39$ episodios), AnB-L 15,1\% $(\mathrm{n}=23$ prescripciones), voriconazol oral $14,5 \%(n=22$ prescripciones $)$ y posaconazol $5,9 \%(n=9$ prescripciones $)$.

\section{Evaluación de las prescripciones}

Del total de prescripciones, $24,3 \%(n=37)$ se asociaron a episodios de EFI probadas, $14,5 \%(\mathrm{n}=22)$ a EFI probables y $30,3 \%(n=46)$ a EFI posible. El resto correspondió a profilaxis $15,8 \%(\mathrm{n}=24)$, EFI descartada $9,9 \%(\mathrm{n}=15)$ y no EFI $5,2 \%(\mathrm{n}=8)$.

\section{Estrategias terapéuticas}

El 55,3\% $(\mathrm{n}=84)$ se indicó en tratamientos empíricos, seguidos de los tratamientos dirigidos $28,9 \%(\mathrm{n}=44)$ y profilaxis $15,8 \%(n=24)$ (Tabla 2$)$. 


\section{Diagnóstico e indicación}

Los pacientes receptores de TPH presentaron el mayor número de indicaciones de antifúngicos $43 \%$ $(\mathrm{n}=65)$ (Tabla 1); la mayoría de los casos correspondió a EFI posible 33,8\% $(n=22)$ con sólo 4,6\% $(n=3)$ de EFI probada siendo el fármaco más utilizado voriconazol iv $40 \%(n=26)$. Esto contrasta con lo observado en pacientes con neoplasias hematológicas en quimioterapia, donde $42,1 \%$ de las indicaciones de antifúngicos $(\mathrm{n}=16)$ se asoció a EFI probadas y $28,9 \%(\mathrm{n}=11)$ a EFI posibles, siendo los fármacos más utilizados anidulafungina y caspofungina en $28,9(\mathrm{n}=11)$ y $15,8 \%$ $(n=6)$, respectivamente.

El consumo más alto de antifúngicos según la clasificación EORTC/MSG, evaluado en días de tratamiento por 100 pacientes día (DOT), fue en indicaciones relacionadas a EFI probada con un DOT probada de 10,54 días, siendo AnB-L y voriconazol iv los fármacos con mayor consumo con un DOT probada AnB-L de 3,15 días y DOT probada voriconazol iv de 3,01 días, respectivamente. Por otro lado, las indicaciones de profilaxis se asociaron a un mayor consumo de antifúngicos con una DOT profilaxis-total de 26,62 días seguido de tratamiento dirigido con un DOT dirigido-total de 11,2 días.

El fármaco sistémico de mayor consumo fue AnBL con un DOT 6,68 días, indicado principalmente en el tratamiento dirigido (DOT dirigido AnB-L 3,15 días) y profilaxis (DOT profilaxis AnB-L 3,15 días).

El fármaco antifúngico oral con mayor consumo fue voriconazol, utilizado principalmente en profilaxis ( DOT profilaxis voriconazol 10,49 días) (Tabla 2).

La duración de las terapias antifúngicas varió dependiendo del fármaco y de la estrategia terapéutica utilizada, siendo los tratamientos dirigidos y las profilaxis las de mayor duración, con una mediana de 15,5 días (rango 1 -

\begin{tabular}{|c|c|c|}
\hline Parámetros & & \\
\hline Pacientes & 79 & \\
\hline Episodios & 152 & \\
\hline Edad (Años; mediana; Rango) & 8 & $(0-19)$ \\
\hline Peso (kg; mediana; Rango) & 27,8 & $(0,9-92)$ \\
\hline \multicolumn{3}{|l|}{ Sexo } \\
\hline Hombres (\%) & 48 & $60,8 \%$ \\
\hline Mujeres (\%) & 31 & $39,2 \%$ \\
\hline \multicolumn{3}{|l|}{ Diagnóstico (\%) } \\
\hline Tumor sólido / episodios & $5 / 6$ & $6,3 \% / 4 \%$ \\
\hline Neoplasia hematológica / episodios & $20 / 38$ & $25,3 \% / 25 \%$ \\
\hline TPH / episodios & $27 / 65$ & $34,2 \% / 43 \%$ \\
\hline TOS / episodios & $12 / 25$ & $15,2 \% / 16 \%$ \\
\hline Otros / episodios & $15 / 18$ & $19 \% / 12 \%$ \\
\hline \multicolumn{3}{|l|}{ Cultivos obtenidos por paciente } \\
\hline Cultivos positivos & 22 & $28 \%$ \\
\hline Candida albicans & 15 & $68 \%$ \\
\hline Candida lusitaniae & 1 & $4,5 \%$ \\
\hline Aspergillus fumigatus & 1 & $4,5 \%$ \\
\hline Aspergillus flavus & 1 & $4,5 \%$ \\
\hline Mucorales & 3 & $14 \%$ \\
\hline Bipolaris sp & 1 & $4,5 \%$ \\
\hline
\end{tabular}

111 días) y 88 días (rango: 1-345 días), respectivamente. Voriconazol oral e intravenoso fueron los tratamientos más prolongados en las estrategias dirigidas, mientras que para la profilaxis fueron posaconazol y voriconazol oral. En las estrategias empíricas, el tratamiento más prolongado fue voriconazol iv con una mediana de 28 días y un rango de 1-92 días.

\begin{tabular}{|c|c|c|c|c|c|c|c|c|c|c|c|c|c|c|c|c|c|c|c|c|c|}
\hline \multirow[t]{2}{*}{ Clasificación } & \multicolumn{3}{|c|}{ Voriconazol iv } & \multicolumn{3}{|c|}{ Voriconazol vo } & \multicolumn{3}{|c|}{ Posaconazol vo } & \multicolumn{3}{|c|}{$\begin{array}{c}\text { Amfotericina B } \\
\text { liposonal }\end{array}$} & \multicolumn{3}{|c|}{ Anidulafungina } & \multicolumn{3}{|c|}{ Caspofungina } & \multicolumn{3}{|c|}{ Total } \\
\hline & $n$ & $\%$ & DOT & $n$ & $\%$ & DOT & $n$ & $\%$ & DOT & $n$ & $\%$ & DOT & $\mathbf{n}$ & $\%$ & DOT & $\mathbf{n}$ & $\%$ & DOT & $\mathrm{n}$ & $\%$ & DOT \\
\hline \multicolumn{22}{|l|}{ Tratamiento } \\
\hline Dirigido & 4 & $9,1 \%$ & 1,23 & 4 & $9,1 \%$ & 3,01 & 2 & $4,5 \%$ & 0,06 & 10 & $22,7 \%$ & 3,15 & 19 & $43,2 \%$ & 3,3 & 5 & $11,4 \%$ & 0,45 & 44 & $28,9 \%$ & 11,2 \\
\hline Empírico & 34 & $40,5 \%$ & 3,21 & 8 & $9,5 \%$ & 2,95 & 0 & $0,0 \%$ & 0 & 7 & $8,3 \%$ & 0,43 & 21 & $25,0 \%$ & 1,97 & 14 & $16,7 \%$ & 0,86 & 84 & $55,3 \%$ & 9,42 \\
\hline Profilaxis & 1 & $4,2 \%$ & 0,01 & 10 & $41,7 \%$ & 10,49 & 7 & $29,2 \%$ & 13,02 & 6 & $25,0 \%$ & 3,1 & 0 & $0,0 \%$ & 0 & 0 & $0,0 \%$ & 0 & 24 & $15,8 \%$ & 26,62 \\
\hline \multicolumn{22}{|l|}{ EORTC } \\
\hline EFI Probada & 4 & $10,8 \%$ & 1,23 & 4 & $10,8 \%$ & 3,01 & 2 & $5,4 \%$ & 0,06 & 10 & $27,0 \%$ & 3,15 & 12 & $32,4 \%$ & 2,6 & 5 & $13,5 \%$ & 0,49 & 37 & $24,3 \%$ & 10,54 \\
\hline EFI Posible & 22 & $47,8 \%$ & 1,61 & 3 & $6,5 \%$ & 0,59 & 0 & $0,0 \%$ & 0 & 3 & $6,5 \%$ & 0,32 & 10 & $21,7 \%$ & 1,04 & 8 & $17,4 \%$ & 0,69 & 46 & $30,3 \%$ & 4,25 \\
\hline EFI Probable & 12 & $54,5 \%$ & 1,6 & 4 & $18,2 \%$ & 2,29 & 0 & $0,0 \%$ & 0 & 2 & $9,1 \%$ & 0,09 & 4 & $18,2 \%$ & 0,46 & 0 & $0,0 \%$ & 0 & 22 & $14,5 \%$ & 4,44 \\
\hline Profilaxis & 1 & $4,2 \%$ & 0,01 & 10 & $41,7 \%$ & 10,49 & 7 & $29,2 \%$ & 13,02 & 6 & $25,0 \%$ & 3,1 & 0 & $0,0 \%$ & 0 & 0 & $0,0 \%$ & 0 & 24 & $15,8 \%$ & 26,62 \\
\hline Descartadas & 0 & $0 \%$ & & 1 & $6,7 \%$ & 0,07 & 0 & $0,0 \%$ & 0 & 2 & $13,3 \%$ & 0,02 & 8 & $53,3 \%$ & 0,72 & 4 & $26,7 \%$ & 0,11 & 15 & $9,9 \%$ & 0,92 \\
\hline No-EFI & 0 & $0,0 \%$ & 0 & 0 & $0,0 \%$ & 0 & 0 & $0,0 \%$ & 0 & 0 & $0,0 \%$ & 0 & 6 & $75,0 \%$ & 0,45 & 2 & $25,0 \%$ & 0,02 & 8 & $5,3 \%$ & 0,47 \\
\hline
\end{tabular}




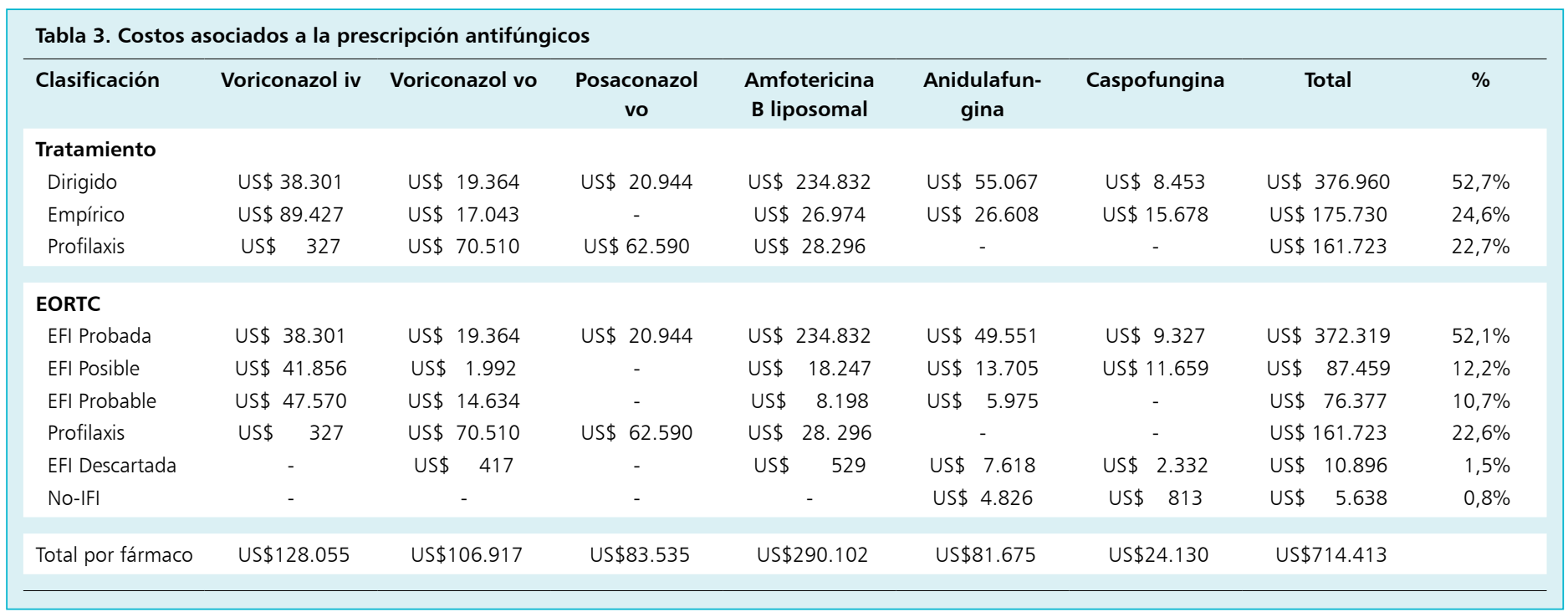

\section{Evaluación económica}

El costo total de los fármacos antifúngicos (Tabla 3) en el período de estudio fue de US \$ 714.413. Con base en la clasificación EORTC / MSG, se observó que 52,1\% del gasto (US \$ 372.319) estaba asociado a prescripciones en EFI probada, 10,7\% (US \$ 76.377) en EFI probable y $0,8 \%$ (US $\$ 5.638$ ) en enfermedad fúngica no-EFI, $12,2 \%$ (US $\$ 87,459$ ) correspondió a prescripciones en EFI posibles y $1,5 \%$ (US \$ 10.896) en EFI descartadas. Finalmente, el 22,6\% restante del gasto antifúngico total de US \$ 161.723 correspondió a indicaciones de profilaxis.

En cuanto a la razón de la indicación, las prescripciones en tratamientos dirigidos generaron el gasto más alto con US \$376.960 (correspondiente a 52,7\% del gasto total), seguidas de tratamientos empíricos y profilácticas con US $\$ 175.730(24,6 \%$ del gasto total) y US \$ $161.723(22,7 \%$ del gasto total), respectivamente.

\section{Discusión}

Este estudio entrega una visión general del uso de fármacos antifúngicos de alto costo en un hospital pediátrico de Chile, cuyo consumo genera un gran impacto presupuestario con un costo de US $\$ 714.413$, que corresponde a $12 \%$ del presupuesto total de farmacia de nuestra institución.

El análisis de los datos obtenidos a partir de esta auditoría y posterior revisión de las fichas clínicas permite inferir que, en la mayoría de los casos, este gasto no está asociado con una mala prescripción, sino con una duración prolongada de las terapias empíricas. Los estudios han demostrado que el gasto asociado a los medicamentos antifúngicos se asocia, principalmente, con el retraso en el de-escalamiento basado en los resultados microbiológicos de los cultivos y en el cambio de la terapia intravenosa a oral. Shan D y cols., por ejemplo, encontraron que en $61 \%$ de los casos tratados con equinocandina no se de-escaló a fluconazol cuando se obtuvieron cultivos y pudo haberse hecho ${ }^{14}$; en nuestro estudio no se evaluó el uso de fluconazol.

Otro problema que genera un uso inadecuado de fármacos antifúngicos, descrito en varias auditorías de fármacos antifúngicos, es la mala adherencia a las guías clínicas. En nuestro estudio, encontramos que la mayoría de las indicaciones empíricas se adhirieron bien a las guías clínicas, tanto nacionales como internacionales (IDSA), para indicar antifúngicos en pacientes con neutropenia febril ${ }^{3,15,16}$ iniciando tratamientos empíricos en pacientes con neoplasias hematológicas o que habían recibido un $\mathrm{TPH}$, con períodos de neutropenia prolongada, uso de antimicrobianos de amplio espectro, antígeno de galactomanano positivo y/o imágenes sugerentes de EFI. En este contexto, los fármacos con mayor uso en tratamientos empíricos, evaluados con DOT, fueron equinocandinas, voriconazol y AnB-L, lo que está relacionado con la epidemiología de la EFI en este grupo de pacientes.

Estudios nacionales han demostrado que la frecuencia de EFI en los niños con neoplasias hematológicas y TPH cursando con neutropenia y fiebre, es de $9 \%$, siendo la candidiasis la EFI más frecuente ${ }^{3}$. Esos datos concuerdan con lo encontrado en el presente estudio donde el microorganismo más aislado fue Candida spp; las equinocandinas son el tratamiento de primera línea en infecciones invasoras por este patógeno en pacientes inmunocomprometidos. 
Por otra parte, observamos diferencias en relación a las indicaciones de antifúngicos en estos dos grupos; los pacientes con TPH tuvieron más indicaciones relacionadas a EFI posibles. Esto puede explicarse principalmente porque los pacientes con TPH tienen varios factores de riesgo que los hacen susceptibles a la EFI como la enfermedad injerto contra hospedero (EICH), los períodos prolongados de neutropenia, las terapias inmunosupresoras, el retardo en la reconstitución inmunológica, el uso de catéteres permanentes y el uso de antimicrobianos de amplio espectro, lo que está asociado a alta mortalidad. El inicio temprano pero adecuado de estas terapias se asocia con mejores resultados clínicos ${ }^{17,18}$.

En segundo lugar, el fármaco más utilizado en los pacientes receptores de TPH fue voriconazol y no equinocandinas como en el caso de las neoplasias hematológicas, lo que puede explicarse por el uso profiláctico de fluconazol en este grupo de pacientes que tiene buena cobertura contra Candida spp pero no contra Aspergillus spp ${ }^{17}$.

Los resultados de nuestro estudio demuestran que, a pesar de existir un control y uso racional de las terapias antifúngicas en el hospital, lo que genera una contención considerable en el gasto asociado con el cuidado del paciente, los costos de estas terapias siguen siendo altos, ocasionando un gran impacto en la institución, principalmente relacionados a fármacos como voriconazol y AnB-L dado su alto costo y prolongada duración de las terapias. En este contexto, aunque un equipo multidisciplinario evalúa la prescripción y el uso de todos los tratamientos antifúngicos, el alto costo de estas terapias hace que el uso inadecuado, aunque sea menor, genere un incremento de gasto. Este uso inadecuado ha sido explicado en base a la dificultad de establecer el diagnóstico y a la alta mortalidad asociada a la EFI, promoviendo el uso excesivo de antifúngicos ${ }^{2-4}$.

En una institución con recursos limitados, el uso inadecuado de antifúngicos tiene un alto impacto en el presupuesto. Lo que se demostró en estudios locales anteriores, donde se estimó que el costo neto atribuible al tratamiento de la aspergilosis invasora fue de \$ 13.274.185 pesos chilenos (US \$ 23.600) .

Dado lo mencionado anteriormente, varios artículos ya han hecho mención a la necesidad del ya mencionado antifungal stewardship. La experiencia internacional indica que la aplicación de estos programas resulta en reducciones significativas en el consumo y en el uso inadecuado, así como en la reducción de $\operatorname{costos}^{4,6,7,10,19-23}$. F. López-Medrano y cols., en su estudio, mostró que la implementación de un programa de antifungal stewardship redujo el gasto anual en antifúngicos, generando un ahorro de US \$ 370.681,78 en comparación con años anteriores. Un programa similar en un hospital de Cambridge, Inglaterra, encontró que la implementación de estos programas logra mejoras significativas en el manejo del paciente y reduce los costos asociados con el uso de antifúngicos, generando un ahorro neto de aproximadamente $£ 180.000$ (US \$ 222.733,80).

Otro hallazgo de nuestro estudio fue el alto gasto asociado con la profilaxis antifúngica, principalmente en pacientes con TPH (US \$ 161.844), debido a que no hay consenso sobre cuándo debe finalizar la profilaxis. Esto es importante en estos pacientes, donde la interrupción de la profilaxis trae un riesgo implícito de tener una EFI, dada su inmunosupresión intensa. Contemplando estos argumentos y la incorporación de nuevos antifúngicos utilizados como alternativas a fluconazol en la profilaxis (posaconazol y voriconazol), creemos que este punto merece una revisión y estudios de costo-efectividad adicionales.

Nuestro estudio tiene varias limitaciones entre las cuales está su carácter retrospectivo que hace difícil encontrar todos los datos en las fichas clínicas de los pacientes. Por otro lado, no encontramos estudios específicos sobre el consumo de antifúngicos en pediatría y/o la implementación de programas de antifungal stewardship. La mayoría de los estudios se realizaron en adultos, lo que hace difícil comparar los resultados, ya que la unidad de consumo utilizada en pediatría es DOT y no la dosis diaria definida (DDD) utilizada en la mayoría de los estudios encontrados. Otra limitación importante es que no se consideró el uso de fluconazol en nuestro estudio, por lo que no se determinaron los tratamientos que se deescalaron y cuánto tiempo tomó para hacerlo en relación con los resultados de los exámenes y cultivos.

\section{Conclusiones}

El consumo de medicamentos antifúngicos genera altos costos correspondiente a $12 \%$ del presupuesto total de farmacia de nuestra institución. El gasto se asoció principalmente a indicaciones en EFI probadas $(52,1 \%$ del gasto de antifúngico), siendo voriconazol (DOT probada voriconazol iv de 3,01) y AnB-L (DOT probada AnBL de 3,15) los con mayor consumo lo que, sumado a su alto costo y días prolongados de terapia, genera un gran impacto en el presupuesto. En este contexto, sumado a la alta complejidad de los pacientes, la necesidad de un equipo multidisciplinario para la evaluación y uso adecuado de los tratamientos, la dificultad para establecer un diagnóstico certero, creemos que es necesario implementar, como ya se ha llevado a cabo en otras partes del mundo, programas de control y gestión enfocados en el uso apropiado de antifúngicos.

Agradecimientos. A los miembros del equipo de farmacia, Unidad de Trasplante de Médula Ósea, Unidad de 
Oncología y Laboratorio Clínico del Hospital Dr. Luis Calvo Mackenna. Además, a la Unidad de Investigación, Facultad de Medicina, Universidad de Chile, Hospital Dr. Luis Calvo Mackenna y María Eliana Maldonado becada de Infectología, Departamento de Pediatría y Cirugía Infantil, Infectología, Universidad de Chile.

\section{Resumen}

Introducción: El incremento de la enfermedad fúngica invasora (EFI) en pacientes inmunocomprometidos ha conducido a la frecuente prescripción de fármacos altamente activos pero de elevado costo económico. Objetivo: Caracterizar el uso de antifúngicos, evaluar su indicación y determinar consumo y costos asociados. Métodos: Estudio descriptivo, retrospectivo, desde enero de 2015 a abril de 2016. Auditoría de prescripciones y revisión de fichas clínicas; cada prescripción se clasificó de acuerdo a si correspondía a una EFI posible, probable o probada. Se calcularon consumos y costos de tratamientos. Resultados: Se auditaron 152 prescripciones de antifúngicos en 79 pacientes. El costo total de los medicamentos antifúngicos fue de US\$ 714.413. El 52,1\% del gasto (US \$372.319) correspondió a indicaciones en EFI probada, $10,7 \%$ (US \$ 76.377) EFI probable, $0.8 \%$ (US \$ 5.638) no-EFI, $12,2 \%$ (US \$ 87.459) EFI posibles y $1,5 \%$ (US \$ 10.896 ) EFI descartada y $22,6 \%$ (US\$ 161.723 ) fue profilaxis. El mayor consumo fue en indicaciones relacionadas a EFI probada con un DOT probada de 10,54 días, siendo anfotericina B liposomal y voriconazol iv los fármacos con mayor consumo con un DOTprobada AnBL de 3,15 y DOT probada voriconazol iv de 3,01. Conclusiones: El consumo de medicamentos antifúngicos genera altos costos correspondiente al $12 \%$ del presupuesto total de farmacia de nuestra institución. El gasto se asoció principalmente a indicaciones en EFI probadas, voriconazol y anfotericina B liposomal los con mayor consumo, lo que sumado a su alto costo y días prolongados de terapia generan un gran impacto en el presupuesto.

\section{Referencias bibliográfícas}

1.- Rabagliati B R, Fuentes L G, Guzmán D A M, Orellana E U, Oporto C J, Aedo C I, et al. Invasive fungal disease in hemato-oncological and hematopoietic stem cell transplantation patients from Hospital Clínico Universidad Católica, Santiago-Chile using revised EORTC/MSG diagnostic criteria. Rev Chilena Infectol 2009; 26(3):212-9. doi: /S071610182009000400002 .

2.- Hamdy R F, Zaoutis T E, Seo S K. Antifungal stewardship considerations for adults and pediatrics. Virulence 2016; 1-15. doi: 10.1080/21505594.2016.1226721.

3.- Santolaya M E, Rabagliati R, Bidart T, Paya E, Guzmán A M, Morales R, et al. Consensus: Rational approach towards the patient with cancer, fever and neutropenia. Rev Chil Infect 2005, 22: S79-113. http://dx.doi.org/10.4067/ S0716-10182005000500001.

4.- Muñoz P, Valerio M, Vena A, Bouza E. Antifungal stewardship in daily practice and health economic implications. Mycoses 2015; 58 Suppl 2:14-25. doi: 10.1111/myc. 12329.

5.- Valerio M, Rodríguez-González C G, Muñoz P, Caliz B, Sanjurjo M, Bouza E, et al. Evaluation of antifungal use in a tertiary care institution: antifungal stewardship urgently needed. J Antimicrob Chemother 2014; 69 (7): 1993-9. doi: $10.1093 / \mathrm{jac} / \mathrm{dku} 053$.

6.- De Souza M C, Dos Santos A G, Reis A M. Drug utilization study of systemic antifungal agents in a Brazilian tertiary care hospital. Int J Clin Pharm 2016; 38 (6): 1398-406.
Epub 2016 Oct 6. DOI: 10.1007/s11096-0160382-6.

7.- Mondain V, Lieutier F, Hasseine L, Gari-Toussaint M, Poiree M, Lions C, et al. A 6-years antifungal stewardship programme in a teaching hospital. Infection 2013; 41 (3): 621-8. doi: 10.1007/s15010-013-0431-1.

8.- Gedik $\mathrm{H}$. The expenditures related to the use of antifungal drugs in patients with hematological cancers: a cost analysis. Clinicoecon Outcomes Res 2015; 7: 537-43. doi:10.2147\%2FCEOR. S92455.

9.- Moreno C M, Del Valle G M, Coria P D. Estudio de costo del tratamiento de la aspergilosis invasora en pacientes oncológicos pediátricos. Santiago. Chile Rev Chilena Infect 2010; 27 (4): 302-7. http://dx.doi.org/10.4067/ S0716-10182010000500002.

10.- Ramos A, Pérez-Velilla C, Asensio A, RuizAntorán B, Floguera C, Cantero M, et al. Antifungal stewardship in a tertiary hospital. Rev Iberoam Micol 2015; 32 (4): 209-13. doi: 10.1016/j.riam.2014.11.006.

11.- De Pauw B, Walsh T J, Donnelly J P, Stevens D A, Edwards J E, Calandra T, et al. Revised definitions of invasive fungal disease from the European Organization for Research and Treatment of Cancer/Invasive Fungal Infections Cooperative Group and the National Institute of Allergy and Infectious Diseases Mycoses Study Group (EORTC/MSG) Consensus Group. Clin Infect Dis 2008; 46 (12): 1813-21. doi: 10.1086/588660.

12.- Polk R E, Fox C, Mahoney A, Mahoney A, Letcavage J, MacDougall C. Measurement of adult antibacterial drug use in 130 US hospitals: comparison of defined daily dose and days of therapy. Clin Infect Dis 2007; 44 (5): 664-70. https://doi.org/10.1086/511640.

13.- Dalton B R, MacTavish S J, Bresee L C, Rajapakse N, Vanderkooi O, Vayalumkal J, et al. Antimicrobial use over a four-year period using days of therapy measurement at a Canadian pediatric acute care hospital. Can J Infect Dis Med Microbiol 2015; 26 (5): 253-8. https://www.ncbi.nlm.nih.gov/ pmc/articles/PMC4644008/pdf/cjidmm-26-253. pdf.

14.- Shah D N, Yau R, Weston J, Lasco T M, Salazar M, Palmer H R, et al. Evaluation of antifungal therapy in patients with candidemia based on susceptibility testing results: Implications for antimicrobial stewardship programmes. . J Antimicrob Chemother 2011; 66 (9): 2146-51. doi: 10.1093/jac/dkr244.

15.- Paganini H, Santolaya M E, Álvarez M, Araña M D J, Arteaga R, Bonilla A, et al. Diagnosis and treatment of febrile neutropenia in pediatric cancer patients. Consensus of the Sociedad Latinoamericana de Infectología Pediátrica. Rev Chile Infect 2011; 28 (Supl 1): 10-38. DOI: 10.4067/S071610182011000400003.

16.- Freifeld A G, Bow E J, Sepkowitz K A, Boeckh M J, Ito J I, Mullen C A, et al. Clinical practice guideline for the use of antimicrobial agents in neutropenic patients with cancer: 2010 update by the Infectious Diseases Society of America. Clin Infect Dis 2011; 52 (4): e56-93. doi: 10.1093/cid/cir073. 
17.- Simms-Waldrip T, Rosen G, NielsenSaines K, Ikeda A, Brown B, Moore T. Invasive fungal infections in pediatric hematopoietic stem cell transplant patients. Infect Dis (Lond) 2015; 47 (4): 218-24. doi: 10.3109/00365548.2014.985709.

18.- Pagano L, Caira M, Candoni A, Offidani M, Fianchi L, Martino B, et al. The epidemiology of fungal infections in patients with hematologic malignancies: the SEIFEM-2004 study. Haematologica 2006; 91 (8): 1068-75.

19.- López-Medrano F, San Juan R, Lizasoain M, Catalan M, Ferrari J M, Chaves F, et al. A non-compulsory stewardship programme for the management of antifungals in a university-affiliated hospital. Clin Microbiol
Infect 2013; 19 (1): 56-61. doi: 10.1111/j.14690691.2012.03891.x

20.- Micallef C, Aliyu S H, Santos R, Brown N M, Rosembert D, Enoch D A. Introduction of an antifungal stewardship programme targeting high-cost antifungals at a tertiary hospital in Cambridge, England. J Antimicrob Chemother 2015; 70 (6): 1908-11. doi: 10.1093/jac/dkv040.

21.- Cook P P, Gooch M. Long-term effects of an antimicrobial stewardship programme at a tertiary-care teaching hospital. Int J Antimicrob Agents 2015; 45 (3): 262-7. doi: 10.1016/j. ijantimicag.2014.11.006.

22.- Reed E E, West J E, Keating E A, Pancholi P, Balada-Llasat J M, Mangino $\mathrm{J}$ E, et al. Improving the management of candidemia through antimicrobial stewardship interventions. Diagn Microbiol Infect Dis 2014; 78 (2): 157-61.doi: 10.1016/j. diagmicrobio.2013.11.012.

23.- Valerio M, Muñoz P, Rodríguez C G, Caliz B, Padilla, Fernández-Cruz A, et al. Antifungal stewardship in a tertiary-care institution: a bedside intervention. Clin Microbiol Infect. 2015 May;21(5):492.e1-9. doi: 10.1016/j. cmi.2015.01.013

24.- Zhao Y J, Khoo A L, Tan G, Teng M, Tee C, Tan B H, et al. Network meta-analysis and pharmacoeconomic evaluation of fluconazole, itraconazole, posaconazole, and voriconazole in invasive fungal infection prophylaxis. Antimicrob Agents Chemother 2015; 60 (1): 376-86. doi: 10.1128/AAC.01985-15. 